\title{
Simulating real-world scenes viewed through ophthalmic lenses
}

\author{
Sergio Barbero ${ }^{1, *}$ and Javier Portilla ${ }^{1}$ \\ ${ }^{1}$ Instituto de Óptica (CSIC), Serrano 121, Madrid, Spain \\ *Corresponding author: sergio.barbero@csic.es \\ Compiled May 16, 2017
}

\begin{abstract}
We present a comprehensive procedure to simulate real-world scenes viewed through ophthalmic lenses. Such method enables to anticipate the effects on image formation of combined undesired optical defects typically found in ophthalmic lenses: blur, distortion and chromatic aberration. Additionally, it helps comparing the expected scenes seen with different lens designs. The procedure is based on the following steps: 1) To calculate distortion and local dioptric matrix associated to a set of different gaze directions; 2) To estimate point-spread-functions (PSF) associated to these matrices; 3 ) To compute the joint action of distortion, chromatic aberration and PSF field on the scenes. We illustrate this procedure with two +5 D spherical lenses: a moderately good performance lens and a highly degradating one. The method is suitable to evaluate ophthalmic lenses in a virtual reality framework. () 2017 Optical Society of America
\end{abstract}

OCIS codes: $\quad$ (330.4595) Optical effects on vision; (330.5370) Physiological optics; (330.7326) Visual optics, modeling; (330.4460) Ophthalmic optics and devices

http://dx.doi.org/10.1364/ao.XX.XXXXXX

\section{INTRODUCTION}

Ophthalmic lenses is the most widespread optical technology used to compensate refractive errors, such as myopia, hypermetropia, astigmatism or presbyopia. However, far from providing a perfect representation of real-world object scenes, spectacles introduce some undesired optical defects that make retinal images differ from the ideal ones. Consequently, it is very relevant to model, as accurately as possible, how real-world scenes are viewed through ophthalmic lenses. This, on the one hand, enables to anticipate the effects on image formation of those undesired optical defects, and on the other hand, to compare them for different lens designs. This is the major motivation of this work.

Due to the rapidly decaying concentration of cones at the retina, the maximum visual acuity is reduced to a small field-ofview (foveal vision). Therefore, voluntarily changing the gaze direction allows imaging at the fovea the part of the scene that we want to see in detail. However, this is not the only eye movement presented in scene scanning. There are also rapid saccades movements as high as 500 degrees per second [1], which are used to fine-tuning the image location at the fovea. After these saccades, the gaze direction remains relatively "fixed" (ignoring nystagmus, drifts and microsaccades) for about 200-300 ms [1]. The visual information is processed only during these fixated periods, because during the rapid saccades the brain blocks the visual process (saccadic supression). Finally, within the visual cortex process, all these "images" are fused providing a unified visual perception of the overall scene.

Considering this knowledge, it is plausible to model scene viewing as a superposition of multiple foveal (or parafoveal) images, each one associated to each fixation between saccades, with a smoothing transition between them. Although not explicitly justified as above, this rationale is behind the proposal made by Barsky et al. [2] some years ago, which they called: scanned foveal image. However, here we prefer to use the term scene image, in order to stress the integrated nature of the visual process during scene viewing .

For a fixed gaze direction, major defects introduced by spectacles are blurring, chromatic aberration, image displacement and distortion (the latter two are intimately connected). Hence, the design of ophthalmic lenses searches for the optimal lens surfaces that minimize these defects when looking through different parts of the lens, within a specific field of view. For this purpose, blur and distortion can be separately analyzed using maps that quantify them $[3,4]$. However, this approach does not provide direct information on how these errors jointly degrade the scene image, as done by scene image simulation.

Furthermore, we propose scene image modeling for, on the one hand, understanding and improving ophthalmic lens designs, and, on the other hand, selecting the best design for a specific need and subject. Inde

ed, in the last decade, the goal of simulating scene images seen through eyeglasses has been tackled by researches in the 
field of computer graphics $[5,6]$, with a special emphasis in progressive addition lenses $[7,8]$. However, the models proposed in the aforementioned works do not provide the way to estimate the relative blur size with respect to the area of the scene. Additionally, they do not consider the radiometric changes associated to the distortion introduced by ophthalmic lenses. These issues (explained in detail in section 2) become relevant if a precise modeling of real scenes degradations is required. That is indispensable, for example, for doing realistic simulations in a virtual reality framework.

This paper aims at providing a clear and justified procedure to simulate scene images through eyeglasses, where the effect of blurring, chromatic aberration and optical distortion is combined, and the relative size of the blurring kernel with respect to scenes dimensions are carefully established.

\section{METHOD}

We first characterize a gaze direction (described by vector $\mathbf{g}$, Figure 1) with angular coordinates $(\theta, \phi)$ having the following meaning. The projection of $\mathbf{g}$ onto planes $X-Z$ (nasal-temporal) and $Y-Z$ (vertical) are $g_{x}$ and $g_{y}$, respectively. Then, $\theta$ and $\phi$ are the angles between direction $Z$ and vectors $g_{x}$ and $g_{y}$, respectively. Therefore, $\theta$ and $\phi$ quantify the angular deviation of gaze direction, in the horizontal and vertical directions, from a primary line of sight along $Z$.

We can define a vector space $\aleph$ containing all possible gaze directions (g) of the naked eye and another vector space $\Im$ with the gaze directions when looking through an ophthalmic lens. Once an object point is fixed (e.g point $a$ at Figure 2), the gaze direction corresponding to each vector space $\left(\mathbf{g}_{a}\right.$ and $\left.\mathbf{g}_{s a}\right)$ are optically linked. The line along the naked gaze direction $\left(\mathbf{g}_{a}\right)$ intersects its associated $\mathbf{g}_{s a}$ (after refraction through the lens) at the object point $(a)$. Therefore, we can define a mapping between both vector spaces: $\mathbf{T}: \aleph \longrightarrow \Im$, or, using angular coordinates between sets: $\mathbf{T}:(\theta, \phi) \longrightarrow\left(\theta^{s}, \phi^{S}\right)$. The smoothness of refractive surfaces used in ophthalmic applications imposes that mapping $\mathbf{T}$ itself is smooth (see a discussion on this at [3]). Additionally, we assume something that occurs in virtually all practical designs, namely, that mapping is invertible (inverse mapping $\mathbf{T}^{-1}$ ). Furthermore, we assume that the inverse mapping itself is smooth too, something that also occurs in practice. This allows for $\mathbf{T}$ and $\mathbf{T}^{-1}$ being safely interpolated, a property that we exploit throughout this paper.

We now define an ideal scene image as that without optical defects, i.e. zero blurring and distortion, being mathematically represented as function of angular coordinates: $I_{0}(\theta, \phi, \lambda)$, being $I_{0}$ the radiance captured by the eye for a specific gaze direction and wavelength $\lambda$. We assume that for the primary line of sight, eyeglasses perfectly correct sphere and cylinder, hence canceling the blur. To mathematically characterize how the scene image is degraded within a range of eye rotations implies to evaluate how the radiance function is transformed between spaces $\aleph$ and $\Im: \mathbf{G}: I_{o}(\theta, \phi) \longrightarrow I_{\mathcal{S}}\left(\theta^{S}, \phi^{S}\right)$.

Although physically it has a different meaning, formally transformation $\mathbf{G}$ is identical to that between the irradiance distribution at the object and the image plane in an incoherent optical system. For this case, the output energy is just the addition of impulse responses to each object point composing the extended source [9]. We just substitute the object point of the classical imaging formula with a gaze direction and get the following superposition integral:

$$
I_{S}\left(\theta^{s}, \phi^{s}, \lambda\right)=\iint_{\mathcal{N}} h\left(\theta^{s}, \phi^{s}, \theta, \phi ; \lambda\right) I_{o}(\theta, \phi ; \lambda) d \theta d \phi,
$$

where $h\left(\theta^{s}, \phi^{s}, \theta, \phi ; \lambda\right)$ is the impulse response for monochromatic incoherent imaging. Within this context we call this function point spread function (PSF).

Eq. 1 has to be evaluated numerically in vector space $\aleph$ by using a uniform sampling at $\aleph:\left(\theta_{j}, \phi_{j}\right), j=1 \ldots m$. However, to obtain it we should find the corresponding non-uniform set at $\Im$ that would map onto $\aleph: \mathbf{T}^{-1}:\left(\theta_{j}^{s}, \phi_{j}^{s}\right) \longrightarrow\left(\theta_{j}, \phi_{j}\right)$, something that is not straightforward. Instead, we adopted a different strategy, namely to evaluate the involved functions on a uniform lattice in $\aleph$, and then resample them according to the inverse mapping $\mathbf{T}^{-1}$, such that the new quantities correspond to a uniform sampling in $\aleph$. It is worth to note that most authors ignore the uniform sampling requirement, evaluating the involved functions in a uniform grid in $\Im$ (e.g. $[5,6,8])$. This translates into a significant error, corresponding to ignoring the scaling factor produced by the Jacobian of $\mathbf{T}$, which represents the variable energy density at different spatial locations due to optical distortion. However, this effect does not affect the PSF field and the optical distortion.

Overall, to simulate the effect of Eq. 1 we propose a computational model comprising the following sequential steps:

1. To choose a uniform grid at $\Im:\left(\theta_{j}^{s}, \phi_{j}^{s}\right), j=1 \ldots m$.

2. To compute the dioptric matrix (R) for each $\left(\theta_{j}^{s}, \phi_{j}^{s}\right)$ : $\mathbf{R}\left(\theta_{j}^{s}, \phi_{j}^{s}\right)$, as explained in section A.

3. To compute the PSF field from the dioptric matrix $\mathbf{R}(\theta, \phi)$, involved in Equation 1 (section B).

4. To obtain a set of $\mathbf{R}(\theta, \phi)$ at a uniform grid of $\aleph$, and repeat the previous steps for three different chromatic channels (section C).

5. A method to compute Eq. 1 once the PSF field is known. This step is accounted for in section D.

\section{A. Dioptric matrix computations}

For each viewing point of the scene we compute the geometrical PSF due only to the spectacles. In this case it is customary to characterize optical aberrations within first-order geometrical optics (i.e., aberrations due to pupil size are ignored [10]) and, consequently, to locally describe them with a linear transformation, performed by the dioptric matrix [11].

To compute the dioptric matrix associated to each viewing point of the scene, the wavefront vergence and the gaze direction are required. Every viewing point (e.g. points $a$ and $b$ in Figure 2) corresponds to a unique gaze direction $\left(\mathbf{g}_{s a}\right.$ and $\left.\mathbf{g}_{s b}\right)$. The distance to the viewing point along the gaze direction determines the vergence of the wavefront emerging from that point. We assume that this distance is known and represented as a function of angular coordinates: $d\left(\theta_{j}^{s}, \phi_{j}^{S}\right)$. It could be directly computed, for instance in a virtual reality framework, by tracing rays and finding their intersections with the object surface scene. Alternatively, there are imaging devices with capability to estimate the distance to the camera of the viewed objects (e.g. [12]). The so captured scenes associate a distance to each pixel in the image (RGB-d pixel format). 
There are several methods available to compute the dioptric matrix from the geometry of the lens and the object distance. Loos et al. [13] proposed tracing localized wavefronts through ophthalmic lenses, particularly progressive lenses. That method is based on applying laborious differential geometry formulae involving curvature data of the lens surfaces and wavefronts [8, 14].

However, in this work we prefer to follow a differential ray tracing method, details of which have been published before by ourselves [3]. We note that the accommodation of the eye is not explicitly considered in this work, but could be easily implemented changing the vertex sphere (see [3]) if that information was known. Indeed, this step would be unavoidable when analyzing mid-near vision scenarios (presbyopic corrections).

\section{B. From dioptric matrix to the PSF}

As explained before, our model depends on angular coordinates that characterize global eye movements. However the shape of the blur (associated to the PSF field of Eq. 1) is an irradiance function that hinges on spatial coordinates at the retina. Therefore, a correspondence between both sets of coordinates is required.

An object seen with a solid angle from the eye has an associated subtended angle measured at the retina. This angle can be computed using the concept of nodal points [15]. Within paraxial approximation a ray coming from the object space forms the same angle with the optical axis when passing through the front and back nodal points. Obviously this implies that the lateral size of the viewing object is reduced at the retina. However, considering angular coordinates, the scene subtends the same angle at object and image (where retina is situated) spaces. This does not happen when the object is seen through eyeglasses, because they introduce an overall magnification (included in the mapping $\mathbf{T}$ ).

Conventional Snellen letter charts, used to measure visual acuity, implicitly assume that a letter could be recognized by a refractive corrected healthy eye if the limbs of the characters subtend a minimum of 1 minute of arc [16]. This value is sustained in theoretical and experimental studies. The theoretical maximum resolution of the human eye, considering the separation of cones at the fovea and the diffraction theory, is about 48 seconds of arc; however, experimental studies lower this value to around 90 seconds of arc (p. 24 [16]). The maximum resolution threshold sets the limit to the admissible spacing between angular coordinates at the retina. For this paper, we used 90 seconds of arc.

Within first-order geometrical optics the PSF takes the form of a uniformly illuminated ellipse (a disc in the absence of cylinder) called defocus blur $[15,17,18]$.

To obtain the defocus blur from the dioptric matrix two steps are required. First, to translate the dioptric matrix into a geometrical ellipse. Second, to convert the values of the semi-axes of the ellipse into angular coordinates. We note here that Kakimoto et al [5] provided a procedure to the first step without clarifying the second one.

We have followed a similar procedure to that of G. Smith [17]. However, whereas Smith started with a defocus defined at the object space, we directly applied the blur at the retina, because the dioptric matrix provides the defocus with respect to the paraxial location within the eye.

As mentioned before, we assume that the eye is emmetropic for the central viewing direction. Therefore, a pencil of rays associated to this central viewing direction and passing through the exit pupil converges to a unique point at the retina given by the focal length $H^{\prime} F^{\prime}$ (see Figure 3). In that case the eigenvalues of the dioptric matrix are equal and equivalent to the required prescription power of the subject.

When looking through different areas of the spectacles the dioptric matrix changes. The inverse of the eigen-values of the dioptric matrix for those gaze directions provide a major $\left(f_{1}\right)$ and a minor focal length $\left(f_{2}\right)$. Let set one of them: $f_{j}$. A marginal ray (passing through the edge of the exit pupil) will cross the optical axis (point G Fig. 3) and intersects the retina at $\zeta_{j}^{\prime}$. This magnitude provides the lateral size of the blur ellipse at the retina for that specific meridian. However, as explained before, we need the angular magnitude of the blur with respect to the back nodal point $\mathrm{N}^{\prime}$. We denote this magnitude: $\psi_{j}=$ $\arctan \left(\frac{\zeta_{j}}{N^{\prime} F^{\prime}}\right)$. Considering similar triangles at Fig. 3 it is easy to see the relation: $\zeta_{j}=\frac{\rho\left(H^{\prime} F^{\prime}-f_{j}\right)}{f_{j}}$. Thus:

$$
\psi_{j}=\arctan \left(\frac{\rho\left(H^{\prime} F^{\prime}-f_{j}\right)}{f_{j} N^{\prime} F^{\prime}}\right) .
$$

In this work we used Gullstrand's eye model $n^{\circ} 1$ [15] for which $\mathrm{H}^{\prime} \mathrm{F}^{\prime}=22.785 \mathrm{~mm}$ and $\mathrm{H}^{\prime} \mathrm{N}^{\prime}=5.73 \mathrm{~mm}$. Additionally, we assumed $\rho=2.5 \mathrm{~mm}$.

Finally, each elliptical PSF is described by a set of three scalars $\left(\psi_{1}, \psi_{2}, \alpha\right)$, where $\left(\psi_{1}, \psi_{2}\right)$ refer to the ellipse's major and minor axis and $\alpha$ is the angle formed by the major axis with the abscissa (Figure 4) which is obtained from the eigenvectors of the dioptric matrix.

\section{Dioptric matrices at $\Im$ space}

Next step is to compute the PSFs on a uniform grid at $\Im$ space with an interval of 90 seconds of arc. The procedure followed here was first to obtain (through non-uniform interpolation) a coarse approximation, on a regular grid in $\aleph$, of mapping $\mathbf{T}$ and the scalars descriptors of the dioptric matrix: $\left(\psi_{1, j}, \psi_{2, j}, \alpha_{j}\right), j=$ $1 . . . m$, and then use a standard uniform interpolation to refine to the final $90 \mathrm{arcsec} /$ pixel interval grid. Given that, due to its symmetry, the ellipse's orientation ranges from 0 to $\pi$, whereas the computed angles from dioptric matrix are in the $\left[\begin{array}{ll}0 & 2 \pi\end{array}\right]$ range. In order to avoid discontinuities between adjacent equivalent angles (e.g., $\pi / 2$ and $3 \pi / 2$ ), we have resampled and interpolated $\cos (2 \alpha)$ and $\sin (2 \alpha)$ instead of $\alpha$ itself. Then, we recover the angle by applying $\alpha=1 / 2 \arctan (\sin (2 \alpha) / \cos (2 \alpha))$.

So far, our analysis has been confined to monochromatic light. However, to take into account chromatic defects we have to evaluate Eq. 1 for different wavelengths and combine the effects. A reasonable and manageable procedure is to consider three wavelengths. We used the three wavelength channels typically used to characterize ophthalmic lenses [19]: Hydrogen (red) C-line, Helium (central) d-line and Hydrogen (blue) F-line with $656.27 \mu m, 587.56 \mu m$ and $486.13 \mu m$ respectively. The associated refractive indexes are denoted with: $n_{C}, n_{d}$ and $n_{F}$. From now on, in a first (rough) approximation we identify these wavelengths with the red, green and blue (RGB) channels used in trichromatic imaging and display devices. A more accurate color representation would imply using many wavelengths to approximate continuous spectral profiles.

\section{Simulating the effect of distortion and space-variant PSF}

Once the PSF field has been computed we can evaluate Eq. 1. There are many methods proposed in computer graphics and image processing literature to compute this imaging equation with a space-variant PSF. A way to proceed is to compute the 
integration kernel from the PSF field (which is not trivial, and rather computationally complex), and then apply some techniques based on convolution and the Singular Value Decomposition (see, e.g., [20, 21]). However these methods can not easily integrate the distortion effect.

An alternative approach, which includes the distortion (mapping T) simultaneously with the blurring, is to directly simulate the aggregation of PSFs, each one centered at the coordinates given by $\mathbf{T}$.

For this purpose, computer graphics researchers have simulated blurred features using the so-called distributed ray tracing [22] techniques. The idea is to trace multiple samples from each pixel/voxel location of the scene, distributed according to the corresponding (previously computed) PSF field. A classical solution is to use a random sampling (see, e.g., [23]). However, other possibilities are open, like to do a uniform deterministic sampling covering the pixel [8]. This latter method is specially suitable for our purposes, because our PSFs, like in [8], are uniform elliptical disks. Instead of actually tracing all those rays, in our case we only need to trace a central ray, and simply apply the geometrical transformation induced by the local dioptric matrix to a normalized circular disk made of uniform samples, defined in the reference image space. Each color component of a pixel in the reference image gives raise to an elliptical cloud of dots in the image space, as indicated in Fig. 4, whose contributions to the final image pixels' values are aggregated. For the reference circular disk we used 197 dots, each mapped and bi-linearly shared by adjoint cells in $\Im$ grid, before integrating them. Additionally we imposed a minimal value of 90 arc-seconds for the width of the ellipse's axes.

Finally, in order to avoid undesired effects of positive magnification (digital zoom), producing unnatural smoothness, we used a reference image with a higher angular sampling rate (60 arcsec/pixel) than the simulation result (90 arcsec/pixel). These sampling rates difference allows for up to $50 \%$ positive magnification free from digital zoom artifacts.

\section{EXAMPLES OF APPLICATION}

We simulated scene image degradation for two different types of $+5 \mathrm{D}$ spherical lenses. To compute the defocus blur in both lenses we assumed a $2.5 \mathrm{~mm}$ pupil radius.

Lens A: The radii of curvature of the front and back surfaces were +56.6 and $+131 \mathrm{~mm}$ respectively. Central thickness was $3 \mathrm{~mm}$. We assumed a CR39 plastic material, with the following refractive indexes: $n_{d}=1.498, n_{F}=1.504$ and $n_{C}=1.4956$ [19]. This design approximately represents the so-called Percival solution [19], which corresponds to the spherical lens that minimizes the mean power error for all gaze directions, leaving some degree of astigmatism. Additionally, the dispersion chromatic power of CR39 is moderate.

Lens B: We selected an extreme flat design (almost planoconvex). The radii of curvature of the front and back surfaces were +114 and $+3903 \mathrm{~mm}$ respectively. As for Lens A, central thickness was $3 \mathrm{~mm}$. For this lens we selected a polycarbonate material, with the following refractive indexes: $n_{d}=1.5855$, $n_{F}=1.5994$ and $n_{C}=1.5799$ [24]. Note that this flat polycarbonate design is not a wearable lens; instead, it has been intentionally designed to produce high polychromatic blur and distortion levels in order to illustrate large image degradations.

For the experiments shown here, we have chosen a simplifying assumption, suitable for a far-sight spectacle design. Namely, we have considered a scene made of far objects. Then, we avoid computing the depth map $d(\theta, \phi)$ that specifies the distance for each view angle. For computation purposes we chose a constant: $d(\theta, \phi)=10^{10}$.

For this purpose, we used here a high-quality photographic outdoors long-distance image: photograph 1C3A6074.CR2, available at www.dpreview.com, with permission from its author, James Carey. In order to remove any traces from the sensor's CFA (color filter array, such as Bayer), we have first decreased the spatial resolution of this image, by locally averaging $2 \times 2$ pixel non-overlapping blocks. We have then cropped a region of $1536 \times 768$ pixels for our reference original, which corresponds to a $25.6^{\circ} \times 12.8^{\circ}$ solid angle, considering a resolution of 60 arcsec/pixel. For obtaining the emmetropic naked-eye image at $90 \mathrm{arcsec} / \mathrm{pixel}$ resolution of Figure 5, we have simply resampled our reference original, by first interpolating $2 \times 2$ and then averaging $3 \times 3$ non-overlapping blocks, resulting in a $2 / 3$ magnification factor. That resulted in a $1024 \times 512$ pixel image. Global scene simulations shown here (Figures 5 and 6) are designed to be visualized from a $45 \mathrm{~cm}$ distance, the eye's primary line of sight being orthogonal to the upper left corner of the image plane.

\section{A. Simulations}

Figures 5 and 6 show how a scene image is seen through Lens A and $\mathrm{B}$, respectively. We exploited the axial rotationally symmetry of the lens examples and plotted only one quadrant of the whole range of eye rotations; i.e $\theta$ and $\phi$ ranging from 0 to $25.6^{\circ}$ and $-12.8^{\circ}$ respectively. The primary line of sight $\left(\theta=0^{\circ}\right.$ and $\phi=0^{\circ}$ ) is located at the top left corner of both images.

The blurring effects (including transverse chromatic aberration) can be more easily perceived in the area close to the down right corner of the scene, to a larger extent when using lens $B$ (Fig. 6). To see more clearly the effects of the scene image degradation, in Figure 7 we zoomed-in two image details located at the right upper and bottom corners of the scene seen through lenses A and B. For comparison purposes we also included the same details as seen with an emmetropic naked eye.

Fig. 7 shows the higher level of blurring of lens B as compared with lens A. Whereas for the latter the number printed over the boat surface (56974), though blurry, can be discriminated, the larger blur introduced by lens B prevents from reading that number. The chromatic effect is more clearly seen at the bottom rigth corner of the scene image. The white strips of the window frame appear as colored bands through both lenses, although the effect is stronger in lens B. Figure 8 helps to understand these effects. It depicts the polychromatic geometrical blurs associated to the gaze directions covering the zoomed-in scene areas of Fig. 7. In both lenses the axial rotational symmetry implies the astigmatism blur has its major axis oriented towards the radial direction. However, lens $B$ generates a larger and more spread astigmatic blur ellipse than that of lens A. The polychromatic geometrical blurs also show how the blueish color are more spread than those closer to red, stressing the role of transverse chromatic aberration when using polycarbonate materials. Indeed, this role is in accordance with previous experimental works [25], which report that the effect of 0.1 prismatic diopter due to transverse chromatic aberration is similar to that due to $0.5 \mathrm{D}$ of cylindrical defocus.

The distortion effects can be more explicitly visualized if a regular mesh image is used instead of a natural scene. This is shown in Figure 9. As expected, lenses A and B generate a distortion that increases radially from center to periphery, much the same as the astigmatic blur [3]. The visual system 
is, nonetheless, more tolerant to distortion than to blur, partly due to neural adaptation and partly to the comparatively lower negative impact of distortion on the visual information flow.

Finally, one can perceive a significant loss of brightness of the simulated images in all examples with respect to the nakedeye reference. Such effect is simply due to the strong positive magnification of the lenses chosen in this article (+5D), which proportionally decreases the irradiance on the retina. However, this phenomenon has usually not a strong visual impact. Under normal circumstances (photopic visual range) a moderate decrease of the global brightness level has quite a small effect on visual perception, thanks to neural adaptation.

\section{DISCUSSION}

In this paper we provide a comprehensive procedure to simulate real-world scenes viewed through ophthalmic lenses. We note that we are just simulating the effects on image formation due to the optical defects induced by ophthalmic lenses, without considering eye's own aberrations. To consider them, one should first measure them and afterwards include their effect in the PSF field computation step.

Such tool is specially useful in the optometry practice because its prediction nature. This, for instance, occurs for off-axis gaze directions in high power monofocal or progressive addition lenses. In those cases, in which chromatic aberration, distortion and blurring are mixed, it is highly important to predict the interaction of all these optical errors in the final scene.

Indeed, our simulation tool could shed light to specific designs trade-offs. For example: should ophthalmic lens designs be lead by a metric that tries to avoid regions having simultaneously low magnification (magnification $<1$ ) and high blurring? [3].

It is quite obvious that the most natural application of our simulation tool is the evaluation of progressive addition lenses (PALs). We have not included any example of it because analyzing PALs involve some, not trivial, adaptions of the general procedure that will be tackled in a future work. In particular, simulating PALs implies a careful analysis on the accommodation changes when looking at different object locations through the lens. The object location modifies both the ellipse blur (due to changes in the object vergence) and the distortion. This effect is included in our procedure by means of $d(\theta, \phi)$ function. However, additionally, the power of the eye itself changes during accommodation because of crystalline lens changes. Such change should be included in our procedure substituting the fixed focal length $\left(H^{\prime} F^{\prime}\right)$ of Eq. 2 with a function $H^{\prime} F^{\prime}=f(d(\theta, \phi))$, depending on the object distance location. This function is the accommodative response of the eye. If unknown, average values for a specific age range could be used [15].

Finally, we note that our procedure is specially suitable to be integrated in a virtual (or augmented) reality framework, providing a fully visual immersion experience. This again would imply extra-work that includes modeling imaging formation through a head-mounted display optical system. Indeed, Hoya Corporation claims to commercialize such a simulation tool (Hoya Vision Simulator [26]), although no openly available demonstrator nor scientific literature is available to make a comparison with our procedure.

\section{A. Acknowledgments}

This work was supported by grant FIS2016-75891-P from the Spanish Ministerio de Economia, Industria y Competitividad.

\section{REFERENCES}

1. K. Rayner, Eye movements in reading and information processing: 20 years of research, Psychol. Bull. 124(3), 372-422 (1998).

2. B.A. Barsky, A.W. Bargteil, D.D. García and S.A. Klein., Introducing vision-realistic rendering, in Eurographics Proceedings, 1-7 (2002).

3. S. Barbero, J. Portilla, Geometrical interpretation of dioptric blurring and magnification in ophthalmic lenses, Opt. Express. 23(10): 13185-13199 (2015).

4. S. Barbero, J. Portilla, On the relation between diopter power and magnification in progressive addition lenses, Ophth. Phy. Opt. 36(44): 421-427 (2016).

5. M. Kakimoto, T. Tatsukawa, Y. Mukai, T. Nishita, Interactive simulation of the human eye depth of field and its correction by spectacle lenses, Comput. Graph. Forum. 26(3), 627-636 (2007).

6. M. Kakimoto, T. Tatsukawa, T. Nishita, An eyeglass simulator using conoid tracing, Comput. Graph. Forum 29(8), 2427-2437 (2010).

7. J. Rodriguez-Celaya, P. Brunet, N. Ezquerra and J.E. Palomar., A Virtual Reality approach to progressive lenses simulation, in XV Congreso Espanol de Informatica Grafica, 1-9 (2005).

8. M. Niesner, R. Sturm, G. Greiner, Real-time simulation and visualization of human vision through eyeglasses on the GPU, in Proceedings of the 11th ACM SIGGRAPH, 195-202 (2012).

9. J.W. Goodman, Introduction to Fourier optics. San Francisco, McGrawHill (1968).

10. D.A. Atchison., Modern optical design assessment and spectacle lenses, Opt. Act. 32(5), 607--634 (1985).

11. J. Alonso, J.A. Gomez-Pedrero, E. Bernabeu, Local dioptric power matrix in a progressive addition lens, Ophthal. Physl. Opt. 17(6), 522-529 (1997).

12. P. Henry, M. Krainin, E. Herbst, X. Ren and D. Fox, RGB-D mapping: Using Kinect-style depth cameras for dense $3 D$ modeling of indoor environments, Int. J. Rob. Res. 31(5): 647-663 (2012).

13. J. Loos, P. Slusallek, H.P. Seidel, Using wavefront tracing for the visualization and optimization of progressive lenses, Comput. Graph. Forum 17(3): 255-265 (1998)

14. O.N. Stavroudis, The optics of rays, wavefronts, and caustics. New York, Academic Press (1972).

15. D.A. Atchison, G. Smith., Optics of the human eye. Edinburgh, Butterworth Heinemann (2000).

16. R.B. Rabbetts, Bennett \& Rabbetts' clinical visual optics. Edinburgh, Elsevier/Butterworth Heinemann (2007).

17. G. Smith, Angular diameter of defocus blur discs, Opt. Vis. Sci. 59(11), 885-889 (1982).

18. C. Chan, G. Smith, R.J. Jacobs., Simulating refractive errors: source and observer methods, Opt. Vis. Sci. 62(3), 207-216 (1985).

19. C. Fowler, Spectacle lenses: theory and practice. Oxford, ButterworthHeinemann (2001).

20. D. Miraut and J. Portilla . Efficient shift-variant image restoration using deformable filtering (Part I), EURASIP J Adv Signal Process 2012(1), (2012).

21. L. Denis, E. Thiébaut, F. Soulez, J. Becker and R. Mourya. Fast Approximations of Shift-Variant Blur. Int. J. Comput. Vision 115(3), 253-278 (2015)

22. R.L. Cook, T. Porter, and L. Carpenter, Distributed ray tracing, in Proceedings of the 11th ACM SIGGRAPH '84, 137-145 (1984).

23. E. Veach, J. Leonidas, and J. Guibas, Optimally combining sampling techniques for Monte Carlo rendering, in Proceedings of the 22nd SIGGRAPH, 419-428 (1995)

24. M.J. Weber, Handbook of optical materials. Boca Raton, CRC Press (2003).

25. C.Y. Tang and W.N. Charman, Effects of monochromatic and chromatic oblique aberrations on visual performance during spectacle lens wear, Ophthal. Physiol. Opt. 12, 340-9 (1992).

26. http://www.hoya-vision-simulator.com/ 


\section{FIGURES}

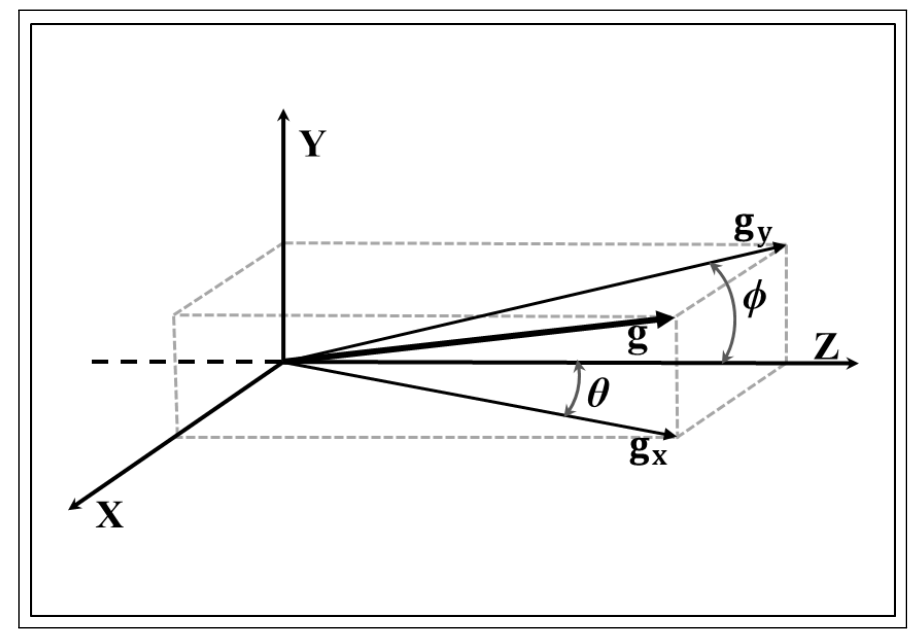

Fig. 1. Gaze direction (g) with respect to the primary line of sight $(z)$ is described by two angular coordinates: $\theta$ and $\phi$.

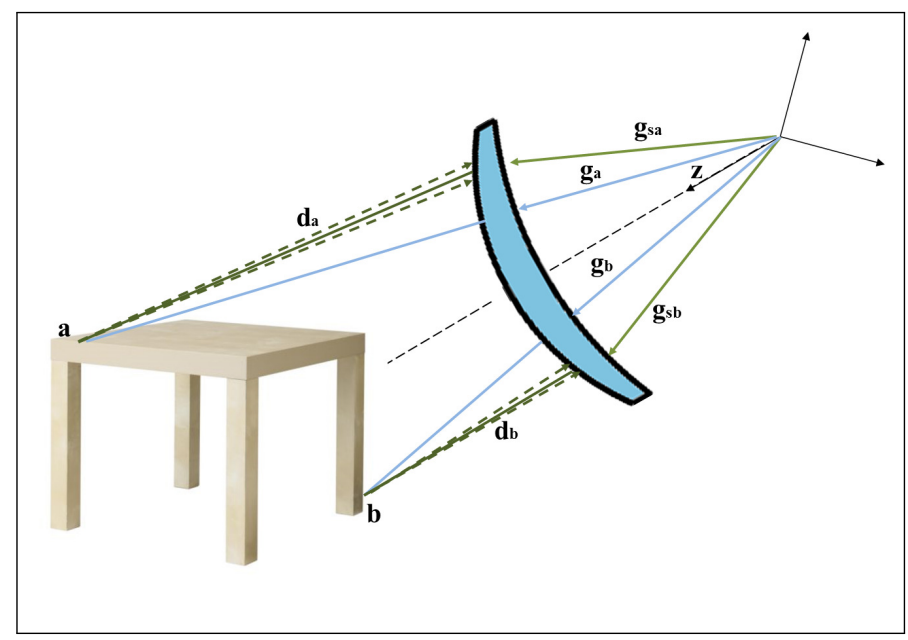

Fig. 2. Scheme showing how the distance from each object point of the scene to the lens changes, thus inducing a different vergence onto the eye.

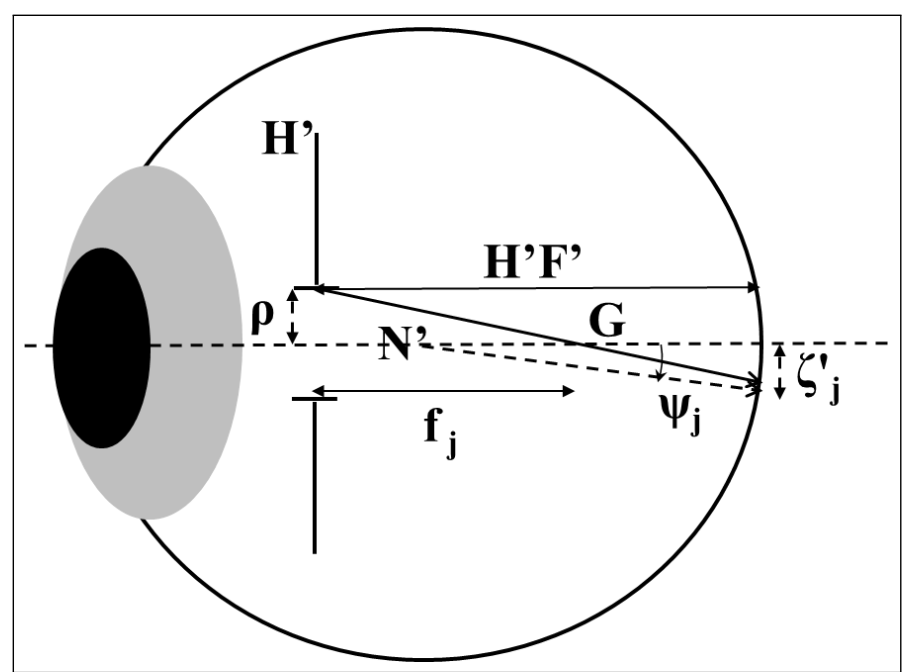

Fig. 3. Elliptical blur size. $\mathrm{H}^{\prime}$ and $\mathrm{N}^{\prime}$ are the back principal and back nodal plane positions, $\rho$ is the radius of the exit pupil and $f_{j}$ a principal focal length.

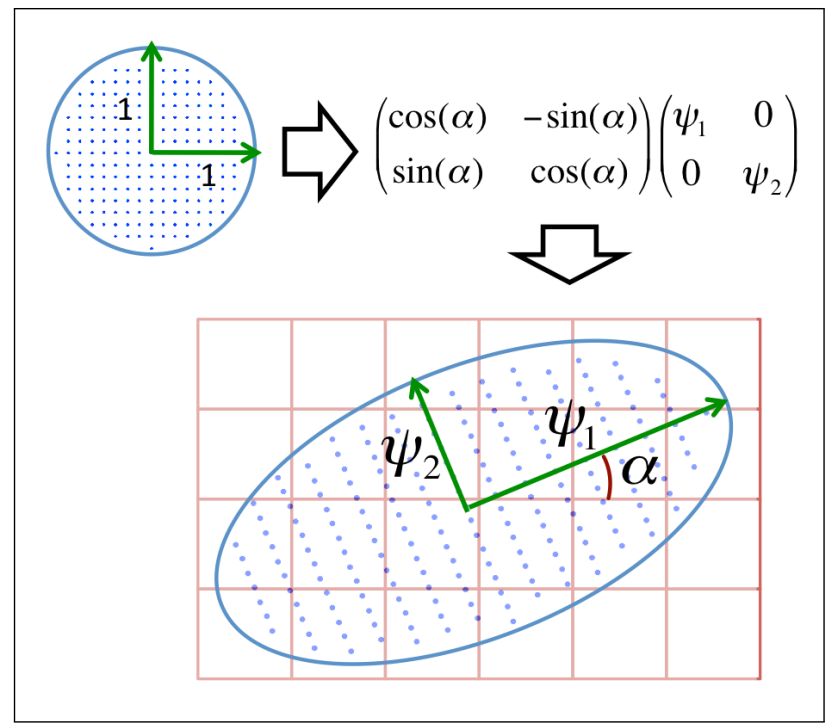

Fig. 4. Generating clouds of elliptical dots to simulate spacevariant blur. 


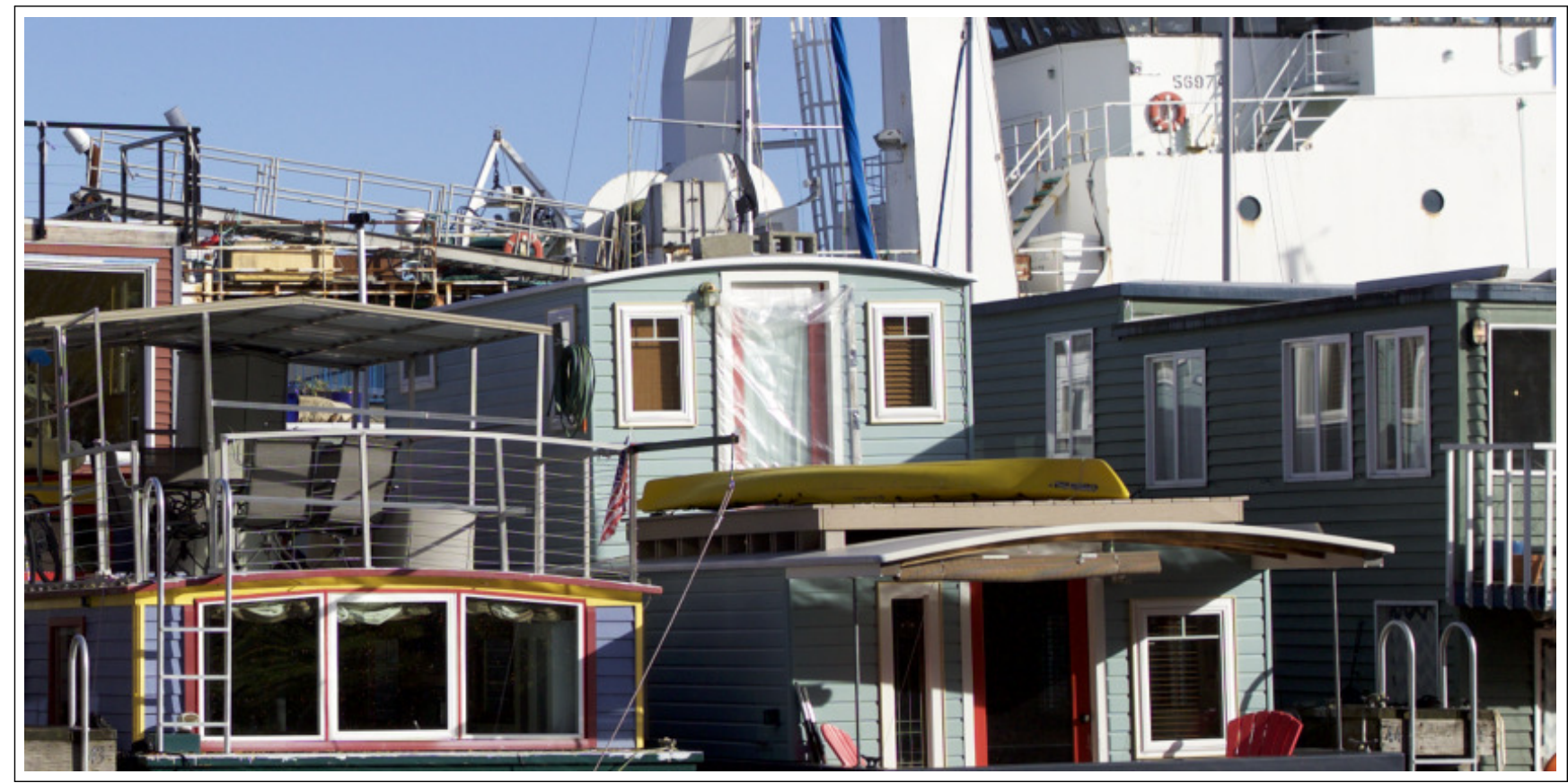

Fig. 5. Simulation of scene image seen with naked eye. Only one quadrant plotted, where the primary line of sight is located in the left top corner. 


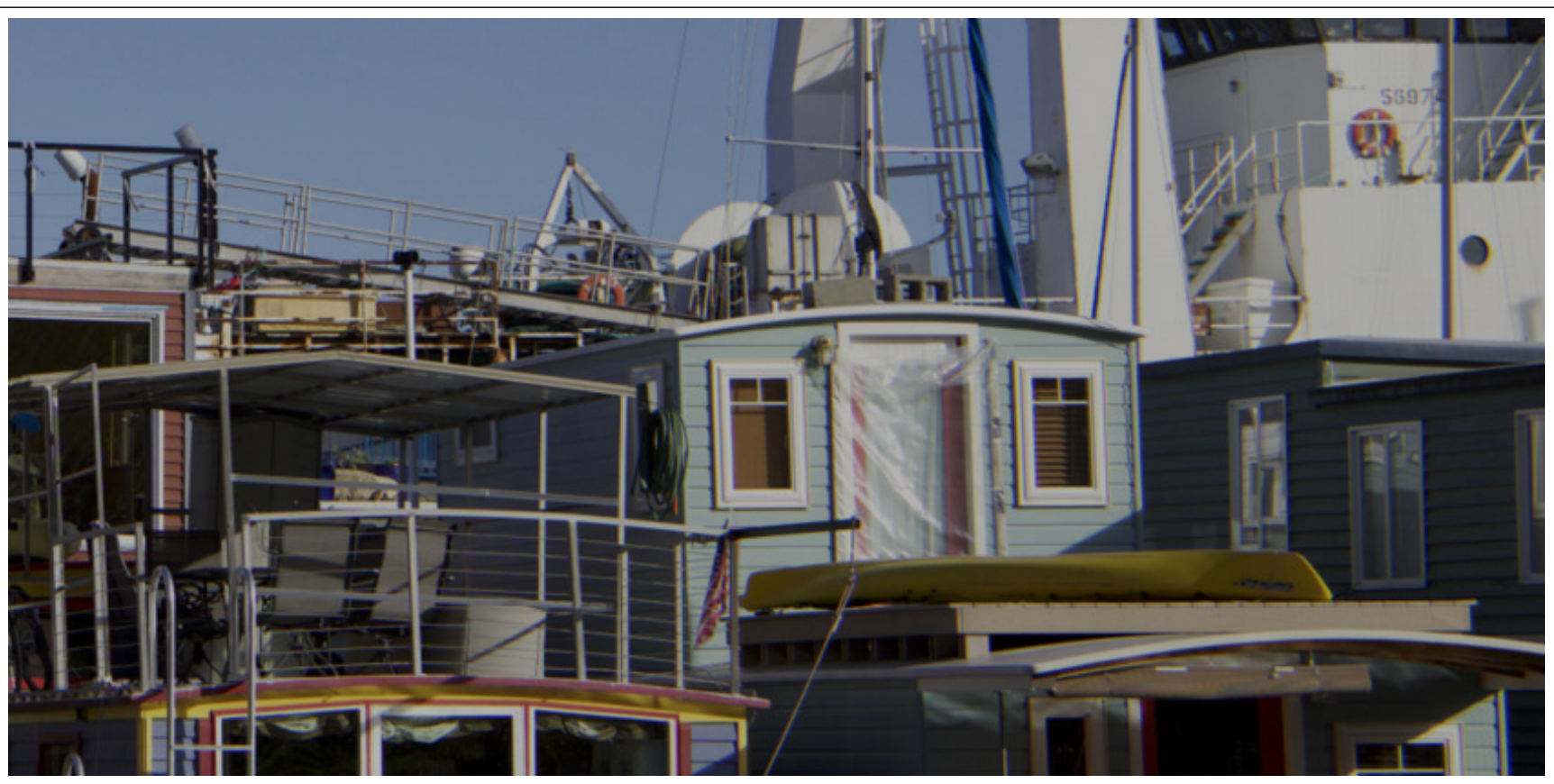

(a)

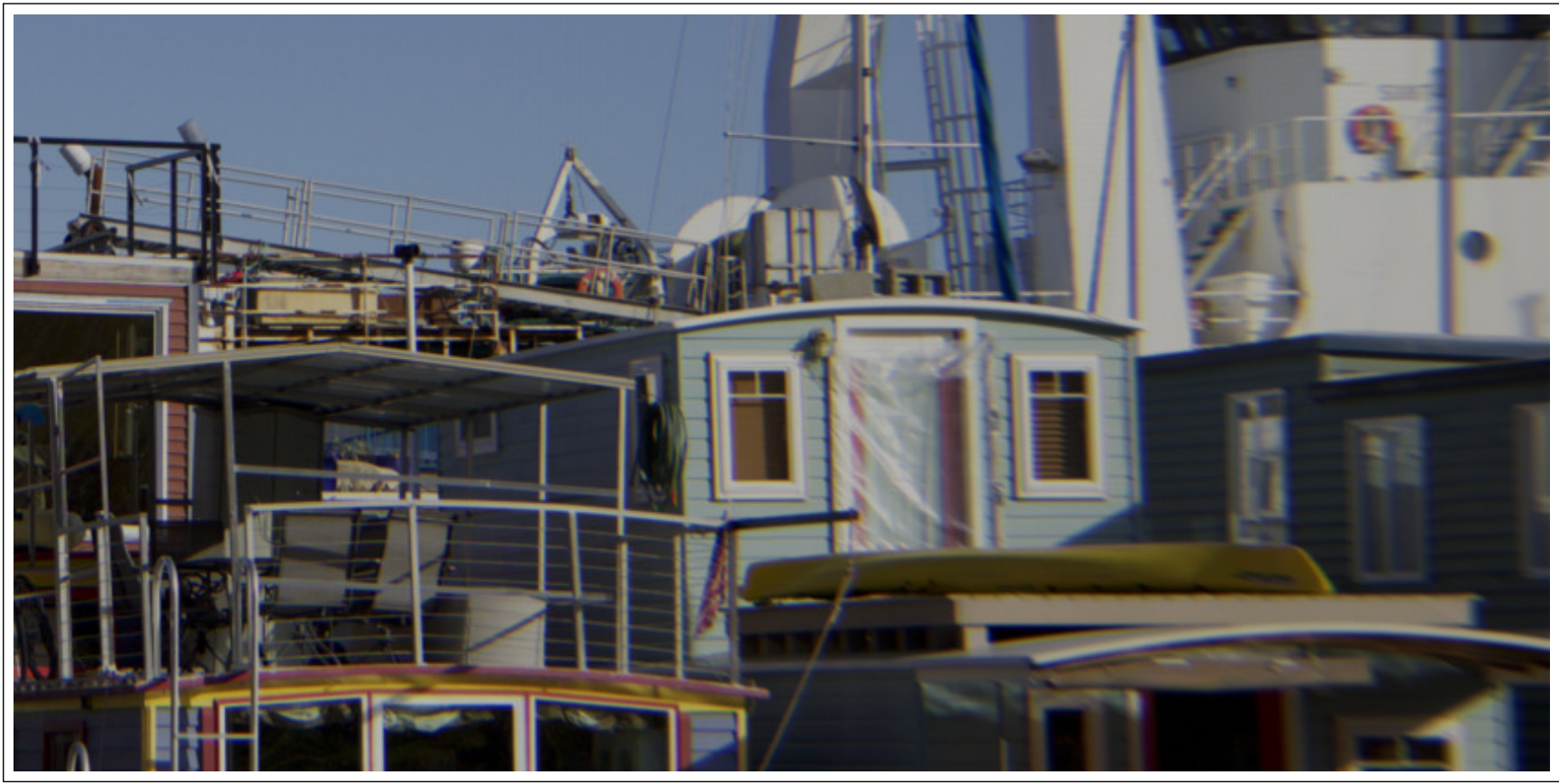

(b)

Fig. 6. Simulation of scene image seen through lenses A (top) and B (bottom). Only one quadrant plotted, where the primary line of sight is located in the left top corner. 


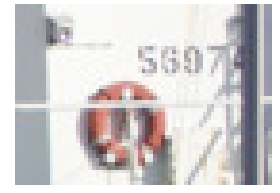

(a)

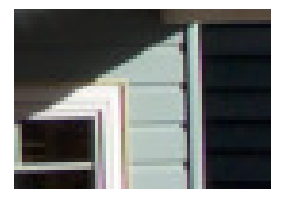

(b)

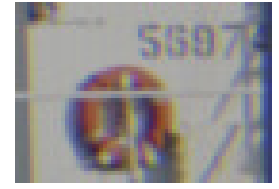

(c)

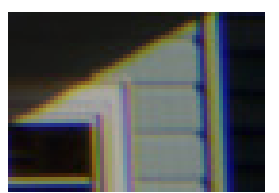

(d)

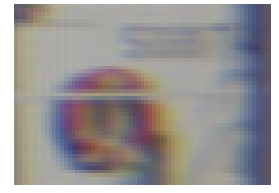

(e)

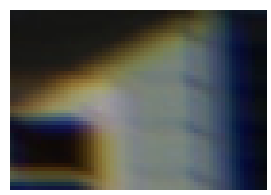

(f)
Fig. 7. Two details of scene image simulation (a)-(b) naked eye; and seen through: (c)-(d) Lens A; (e)-(f) Lens B.

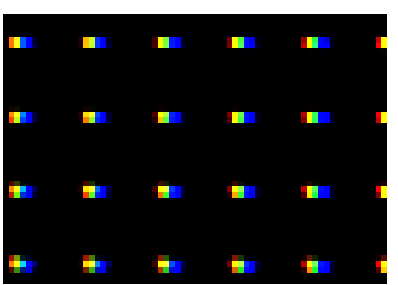

(a)

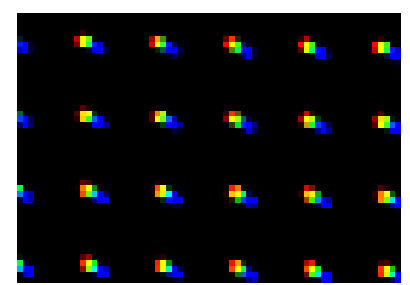

(b)

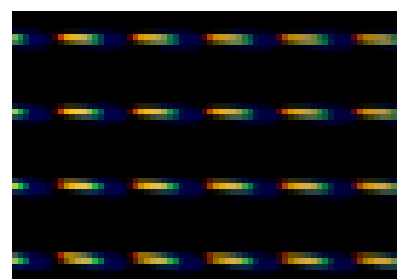

(c)

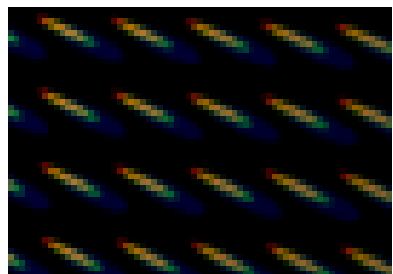

(d)
Fig. 8. Polycromatic PSFs for right top (upper image) and bottom (lower image) details of overall scene image. (a)-(b) Lens A; (c)-(d) Lens B.

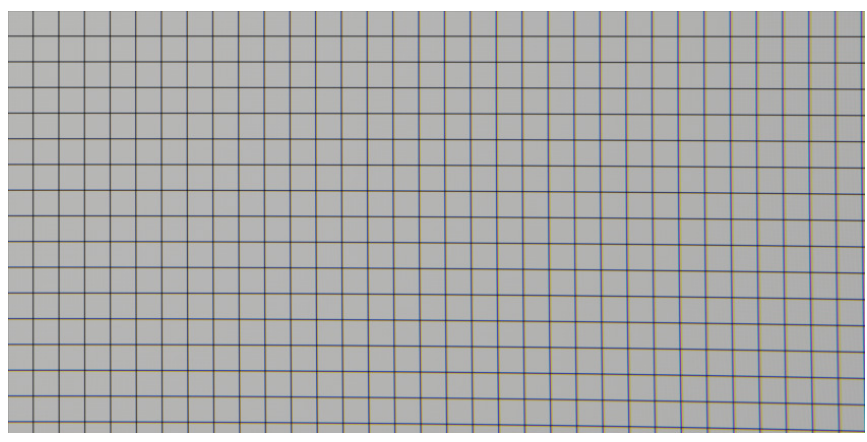

(a)

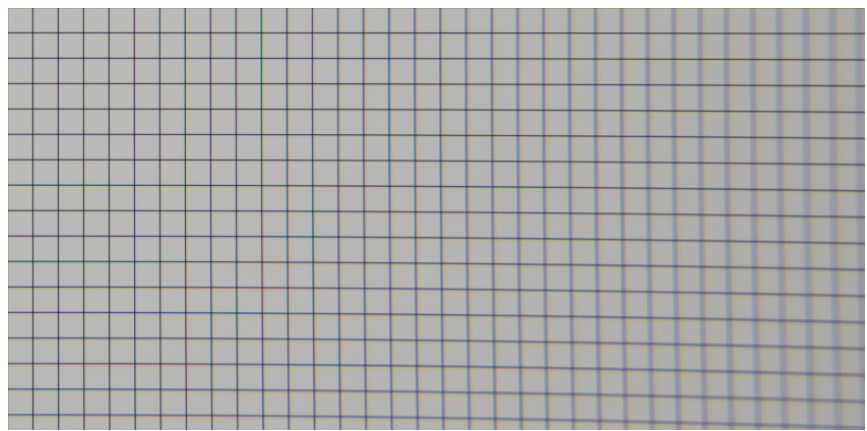

(b)

Fig. 9. Mesh seen through: (a) Lens A; (b) Lens B. Only one quadrant plotted, where the primary line of sight is located in the left top corner. 